ARTICLE

\title{
Diffusive-like redistribution in state-changing collisions between Rydberg atoms and ground state atoms
}

\author{
Philipp Geppert (10 ${ }^{1}$, Max Althön ${ }^{1}$, Daniel Fichtner ${ }^{1} \&$ Herwig Ott (1D ${ }^{1 凶}$
}

Exploring the dynamics of inelastic and reactive collisions on the quantum level is a fundamental goal in quantum chemistry. Such collisions are of particular importance in connection with Rydberg atoms in dense environments since they may considerably influence both the lifetime and the quantum state of the scattered Rydberg atoms. Here, we report on the study of state-changing collisions between Rydberg atoms and ground state atoms. We employ high-resolution momentum spectroscopy to identify the final states. In contrast to previous studies, we find that the outcome of such collisions is not limited to a single hydrogenic manifold. We observe a redistribution of population over a wide range of final states. We also find that even the decay to states with the same angular momentum quantum number as the initial state, but different principal quantum number is possible. We model the underlying physical process in the framework of a short-lived Rydberg quasi-molecular complex, where a charge exchange process gives rise to an oscillating electric field that causes transitions within the Rydberg manifold. The distribution of final states shows a diffusive-like behavior.

\footnotetext{
${ }^{1}$ Department of Physics and Research Center OPTIMAS, Technische Universität Kaiserslautern, Kaiserslautern, Germany. ${ }^{凶}$ email: ott@physik.uni-kl.de
} 
T he understanding of collisions between Rydberg atoms and ground state atoms has a long history and dates back to seminal work done by Fermi ${ }^{1}$. Today, such processes are important for low-temperature plasma physics ${ }^{2}$, astrophysical plasmas $^{3}$, and ultracold atom experiments, which have found in Rydberg physics a perfect match to explore ultracold chemistry and many-body physics: On the one hand, the high control over the internal and external degrees of freedom in an ultracold atomic gas enables the study of new phenomena in the field of Rydberg physics, such as Rydberg molecules ${ }^{4}$, Rydberg blockade ${ }^{5}$, Rydberg antiblockade 6,7 , and coherent many-body dynamics ${ }^{8}$. On the other hand, the same control can now be used to study established processes in a detailed fashion, thus unraveling the underlying microscopic physical mechanisms. This way, the stateresolved study of inelastic collisions and molecular decay processes involving Rydberg atoms has become possible.

Collisions between a Rydberg atom and a ground state atom can have several possible outcomes. Here, we are interested in such collisions of both partners, where the Rydberg atom undergoes a transition to a lower-lying state, while the excess energy is converted into kinetic energy of the atoms. Such collisions have been studied in detail by Schlagmüller et al. ${ }^{9}$. They are important for the understanding of recombination processes in plasmas, for the quantitative understanding of inelastic processes in Rydberg gases ${ }^{10}$ and the decay dynamics of ultralong-range Rydberg molecules (ULRMs).

The microscopic details of such a collision involve the physics of ULRMs ${ }^{4}$, where s- and p-wave scattering between the Rydberg electron and the ground state atom determine the potential energy landscape at large internuclear distances. At short internuclear distances, however, the covalent molecular binding mechanisms take over and dominate the molecular dynamics. The total scattering process therefore probes the potential energy landscape at all internuclear distances. Thus, the understanding of such a process needs the modeling of both the ultralong-range potential energy landscape as well as that at short internuclear distances.

An experimental in-depth study requires the state-selective detection of the reaction products. Only then, it is possible to access branching ratios and selection rules and one can compare the experimental outcome to effective theoretical models. Magnetooptical trap recoil ion momentum spectroscopy (MOTRIMS) $)^{11-22}$ is such a technique, which has been used to perform momentum spectroscopy of atomic and molecular processes with high resolution. Inspired by the MOTRIMS technique, we have developed a high-resolution momentum microscope, which enables the study of inelastic processes involving Rydberg atoms.

Here, we use this technique to investigate inelastic collisions between Rydberg atoms and ground state atoms. In order to increase the collision rate, we excite vibrational states of ULRMs with principal quantum numbers between $n=20$ and $n=27$ as initial state and observe the subsequent dynamics.

\section{Results}

Ultralong-range Rydberg molecules. The interaction of a ground state atom and a Rydberg atom at large internuclear distances is mediated by low-energy scattering between the Rydberg electron and the ground state atom, also denoted as perturber atom. For rubidium, the potential energy at separations larger than the extent of the electronic wave function is given by the energy levels of an isolated Rydberg atom, $E_{n l} \propto-1 /\left(n-\delta_{l}\right)^{2}$, where $n$ is the principal quantum number. The quantum defect $\delta_{l}$ causes a significant splitting of the potential energies only for angular momentum quantum numbers $l \leq 2$. One therefore has to distinguish between energetically isolated low- $l(S, P, D)$ states with

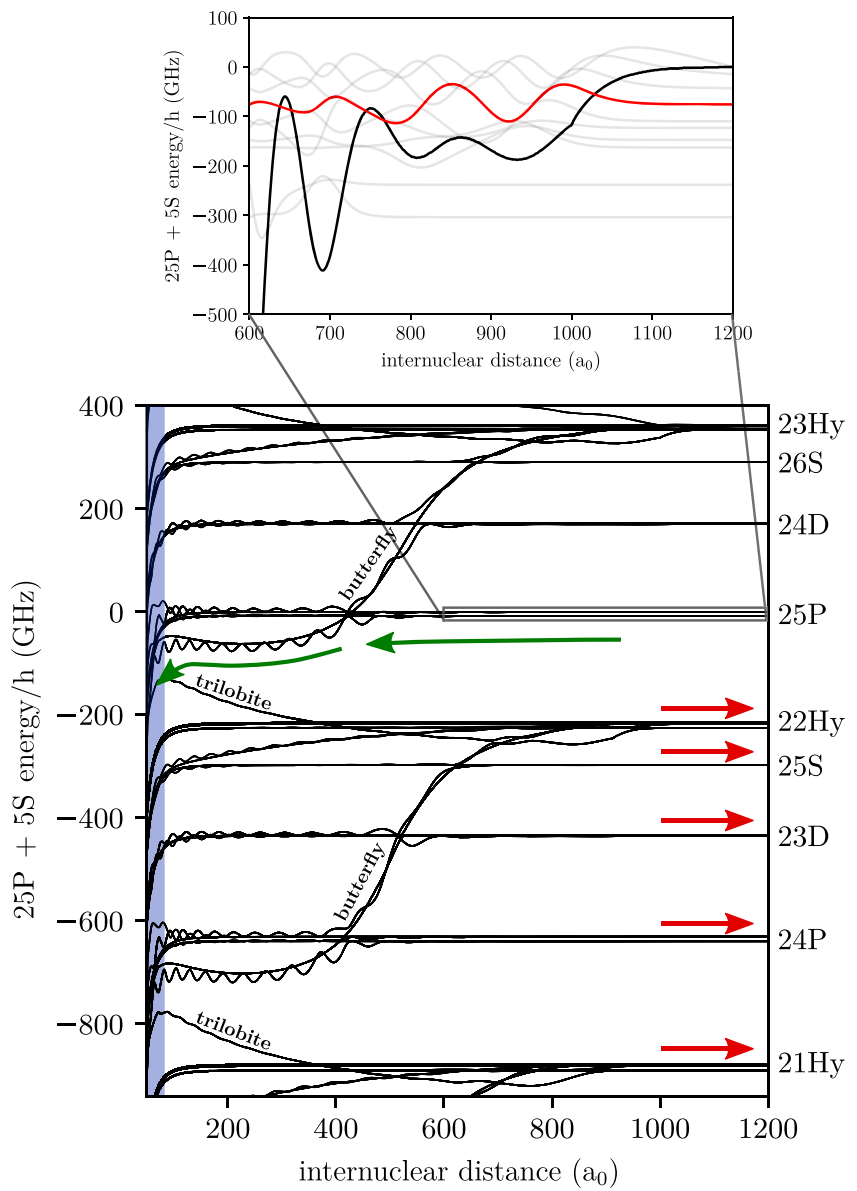

Fig. 1 Adiabatic potential energy curves (PECs) of rubidium ULRMs in the vicinity of the 25P-state. The annotations to the right denote the terms of the asymptotic free Rydberg states. Starting point of our studies is the preparation of rubidium ultralong-range Rydberg molecules (ULRMs), which are bound vibrational states supported by the outermost potential wells at internuclear distances of up to $1000 a_{0}$. The inset shows a zoom into the ultralong-range part of the potential energy landscape with a selection of vibrational wavefunctions (gray curves). Here, we specifically excite vibrational states near the atomic resonance as highlighted in red in the particular case of a 25P-ULRM. As time evolves, the ground state atom tunnels toward the ionic core (green arrows), following the $R^{-4}$-interaction dominated PECs (blue shaded area) up to the region, where short-range molecular couplings are dominant. The red arrows indicate possible outcomes of a state-changing collision, where the release energy is translated into kinetic energy. Details of the calculation of such PECs are provided in the "Methods" section.

significant quantum defects and high- $l$ hydrogenic manifolds. For smaller internuclear distances, the scattering interaction between the Rydberg electron and the ground state atom leads to oscillatory potentials, which support molecular states. The potential energy landscape is shown in Fig. 1. We employ these so-called ULRMs with bond lengths of $\approx 900 a_{0}$ as starting point for our measurements and restrict ourselves to the regime of low principal quantum numbers owing to the $n^{-6}$ scaling of the outer wells' depths ${ }^{23}$. As initial state, we chose molecular states near the atomic Rydberg P-resonance (see inset of Fig. 1), which are excited using a three-photon transition. For a brief introduction to ULRMs, see Methods.

State-changing collisions. The excited molecular states are only weakly bound and the wave function extends over several wells 
up to the inner part of the potential landscape. As a consequence, there is a finite probability for the ground state atom to tunnel toward the ionic core of the Rydberg atom. This way, we mimic an inelastic collision between a free ground state atom and a Rydberg atom, up to a vanishingly small mismatch in energy, which stems from the binding energy of the Rydberg molecule. In other words, the outcome of the collision remains unaffected by the presence of ULRMs. However, unlike initially free atoms, they are capable of mediating collisions with well-defined starting conditions, which makes them an ideal test bed to systematically study the dynamics of inelastic collisions in both a controlled and precise way.

The molecular dynamic is initially dominated by the lowenergy scattering between the electron and the ground state atom $V_{p}(|\boldsymbol{r}-\boldsymbol{R}|)$ (second term in Eq. (8)) and the ion-neutral polarization potential $V_{\mathrm{c}, \mathrm{g}}(R) \propto R^{-4}$ (Eq. 9). In case of alkali atoms, $V_{p}(|\boldsymbol{r}-\boldsymbol{R}|)$ shows a prominent attractive feature, the socalled butterfly potential ${ }^{24-26}$, see Fig. 1 . For our initial states, there is a finite probability of adiabatically following the butterfly potential energy curve (PEC), which accelerates the ground state atom toward the ionic core. At shorter internuclear distances, $V_{c}$, $\mathrm{g}(R)$ takes over and further accelerates the collision process (blue shaded area in Fig. 1). Up to this point, the dynamics can be considered as understood. For even shorter internuclear distances, the ionic core directly interacts with the ground state atom and the Rydberg electron becomes a spectator. As we will detail later on, it is this short-range physics, which is mainly responsible for two distinct processes. The first one is associative ionization, which reads for the case of rubidium

$$
\mathrm{Rb}^{*}+\mathrm{Rb} \rightarrow \mathrm{Rb}_{2}^{+}+\mathrm{e}^{-}+\Delta E_{\mathrm{b}},
$$

where $\Delta E_{\mathrm{b}}$ is the release energy due to the chemical bond of the molecular ion. The second one is a state-changing collision, resulting in an exoergic reaction

$$
\mathrm{Rb}^{*}\left(n_{\mathrm{i}}, l_{\mathrm{i}}\right)+\mathrm{Rb} \rightarrow \mathrm{Rb}^{*}\left(n_{\mathrm{f}} \leq n_{\mathrm{i}}, l_{\mathrm{f}}\right)+\mathrm{Rb}+\Delta E,
$$

where the indices $\mathrm{i}$ and $\mathrm{f}$ denote the initial or final state, respectively. $\Delta E$ is the released energy, which is transformed into kinetic energy of the ground state atom and the Rydberg atom.

In the present work, we address this second type of collisions by directly measuring the momenta of the Rydberg atoms using high-resolution state-resolved momentum spectroscopy. This method enables a clear identification of the final states (sketched as red arrows in Fig. 1) and makes it possible to investigate the distribution of population after the collision.

Recoil-ion momentum spectroscopy. To measure the momentum distributions of Rydberg atoms with high resolution, we have adapted the MOTRIMS technique $\mathrm{e}^{11-22}$, included an optical dipole trap, and implemented a so-called reaction microscope. The image at the top of Fig. 2 shows a three-quarter section CAD drawing of the essential parts of our experimental apparatus.

Each experimental sequence starts with the trapping of precooled ${ }^{87} \mathrm{Rb}$ atoms in a three-dimensional magneto-optical trap (3D MOT). The atoms are then transferred to a crossed optical dipole trap with a wavelength of $1064 \mathrm{~nm}$ (illustrated as red beams in Fig. 2) and trapping frequencies of $\omega_{\mathrm{x}}=2 \pi \times$ $2.8 \mathrm{kHz}, \omega_{\mathrm{y}}=2 \pi \times 1.4 \mathrm{kHz}$, and $\omega_{\mathrm{z}}=2 \pi \times 3.1 \mathrm{kHz}$. After a short evaporation, the sample consists of more than $3 \times 10^{4}$ atoms, prepared in the $\left|5 S_{1 / 2}, F=1\right\rangle$ ground state, with a temperature of $\approx 100 \mu \mathrm{K}$ and a peak density of $1.9 \times 10^{13}$ atoms $/ \mathrm{cm}^{3}$.

Rydberg states $n \mathrm{P}_{3 / 2}$ with principal quantum numbers $n$ between 20 and 27 are addressed via an off-resonant threephoton transition employing the $5 \mathrm{P}_{3 / 2}$ and $5 \mathrm{D}_{5 / 2}$ states as intermediate states. The radiation at $780 \mathrm{~nm}$ (first step), $776 \mathrm{~nm}$
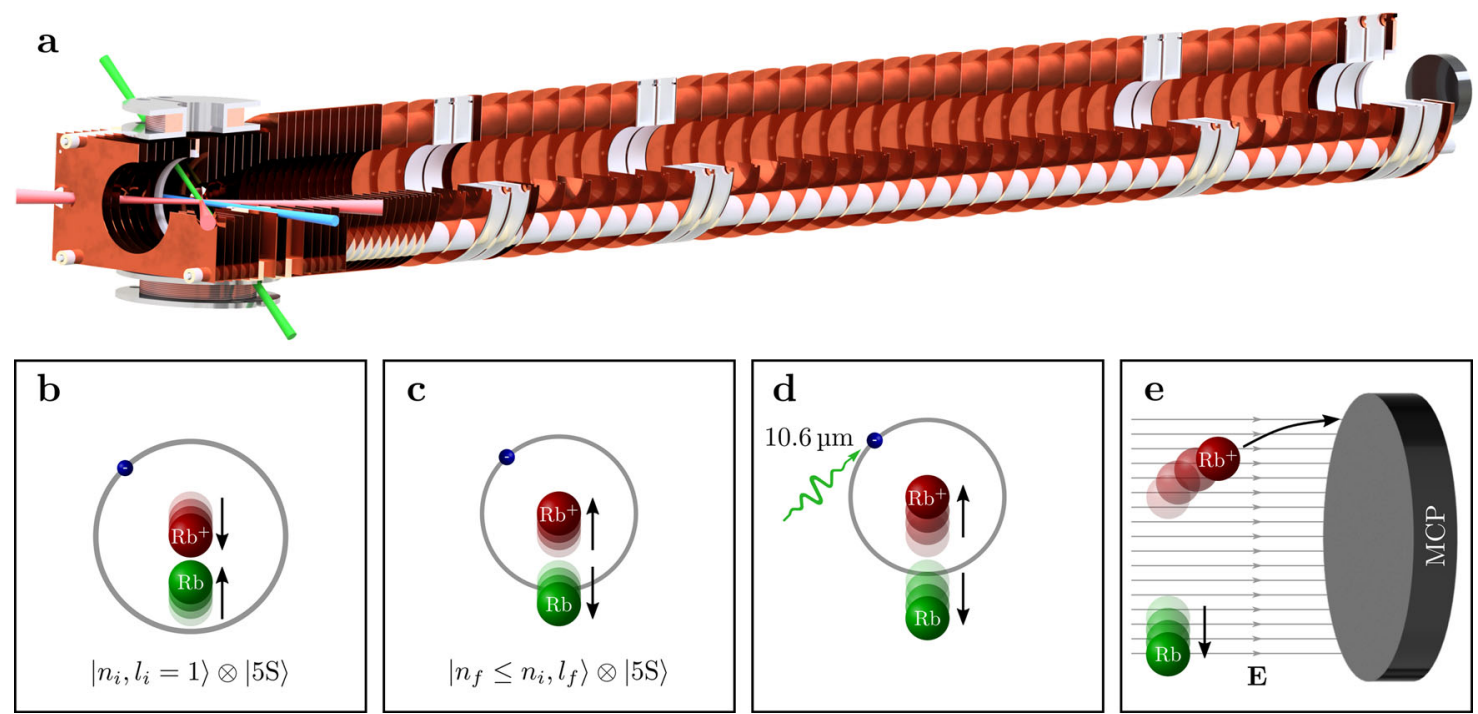

Fig. 2 Recoil-ion momentum spectrometer and experimental sequence. a CAD drawing of the experimental setup in three-quarter section view. Lasercooled ${ }^{87} \mathrm{Rb}$ atoms are trapped in a crossed optical dipole trap (red beams) and excited by a three-photon transition (depicted as blue beam) to a Rydberg state. By using a high-power $\mathrm{CO}_{2}$ laser pulse (green beam), the Rydberg atoms are photoionized efficiently. The ions then follow two subsequent homogeneous electric fields and traverse a field-free drift tube before hitting the position- and time-sensitive detector. This method allows the measurement of momentum distributions of initially neutral particles with high resolution. The lower panel shows a sketch illustrating the state-changing collision process and the experimental procedure to measure the resulting momenta. $\mathbf{b}$ The experiment starts with the photoassociation of an ULRM, where the initial state of the Rydberg electron (blue) is $\left|n_{\mathrm{i}}, l_{\mathrm{i}}=1\right\rangle$. Subsequently, the Rydberg core (red) and the neutral atom (green) approach each other. c During the inelastic collision, the Rydberg electron changes its state (final principal quantum number $n_{\mathrm{f}}$ is less than or equal to $n_{\mathrm{i}}$ ). The final angular momentum quantum number $I_{f}$ may be any value between 0 and $\left(n_{f}-1\right)$. The release energy is apportioned equally between the Rydberg atom and the perturber atom, which fly into opposite directions due to the momentum conservation. $\mathbf{d}$ The Rydberg atom is photoionized by a $\mathrm{CO}_{2}$ laser pulse. e Electric fields guide the ionic core toward the detector without changing the transverse momentum. From the point of impact the momentum of the Rydberg atom can be inferred. 
(second step), and $1280-1310 \mathrm{~nm}$ (third step) is provided by frequency-stabilized diode laser systems. While two excitation lasers are applied from the same direction, the third one is counterpropagating (depicted as blue beam in Fig. 2). The corresponding detunings amount to $\delta_{5 \mathrm{P}}=-60 \mathrm{MHz}$ and $\delta_{5 \mathrm{D}}=$ $+45 \mathrm{MHz}$, respectively. As the photon energy of the dipole trap beams is sufficient to photoionize atoms in the $5 \mathrm{D}_{5 / 2}$ state (photoionization cross section $\gtrsim 17 \mathrm{Mb} \approx 0.6 \mathrm{a}_{0}^{2}$ ), the dipole trap is switched off prior to the $1 \mu$ s long excitation pulse. Subsequently, the atoms are recaptured, such that we can perform up to 100 experiments per sample without loosing too much ( $\lesssim 25 \%)$ density.

At the bottom of Fig. 2, we illustrate the microscopic physical processes. Starting with the photoassociation of ULRMs, we wait for a total of $2 \mu$ s during which the inelastic collisions take place and the Rydberg atom changes its state (Fig. 2a, b). Since the final states are energetically lower than the initial state, the release energy is translated into kinetic energy, which is shared by the Rydberg atom and the ground state atom. Due to momentum conservation, both constituents move into opposite directions. Subsequently, the Rydberg atoms are photoionized by a short pulse from a high-power $\mathrm{CO}_{2}$ laser (Fig. 2c). With a photoionization cross section of tens of megabarns ${ }^{27,28}$ the ionization process is very efficient. The recoil momentum caused by the photoionization is two orders of magnitude smaller than the typical momenta of the investigated processes, such that the created ion has, in good approximation, the same momentum as the Rydberg atom. The ion then follows two sections of homogeneous electric fields and traverses a drift tube with zero electric field before hitting a position and time-sensitive microchannel plate delay-line detector (Fig. 2d). This configuration is referred to as Wiley-McLaren spectrometer ${ }^{29}$ that, in particular, provides space and time focusing of the ions, i.e., ions with the same momentum hit the detector at the same position and the same time, independent of their initial position in the trap. As a result, we are able to measure momentum distributions of initially neutral atoms with resolutions better than $0.1 \hbar / a_{0}$ depending on the chosen electric fields.

Momentum spectroscopy of state-changing collisions. The outcome of our experiments is two-dimensional momentum distributions as shown in Fig. 3 for the 25P-state. A larger part of the ions accumulates at the center, where the transverse momentum is close to zero. These ions stem from photoionized ULRMs which have not undergone a state-changing collision or from facilitated off-resonantly excited Rydberg atoms ${ }^{6}$. Around the center, two concentric circular structures are visible. The circular shape arises from a projection of a three-dimensional spherical shell in momentum space onto the surface of the detector. The sharp boundaries of the circles thereby correspond to momentum vectors perpendicular to the spectrometer axis. As the initial state is well defined, the energy differences $\Delta E$ to each of the lower-lying states and hence the momenta $p$ of the $\mathrm{Rb}^{+}$ ions with the mass $m_{\mathrm{Rb}}$ can be calculated using

$$
p=\frac{\hbar \sqrt{2 m_{\mathrm{Rb}} \Delta E}}{a_{0} E_{\mathrm{H}} m_{\mathrm{e}}},
$$

where $\hbar$ denotes the reduced Planck constant, $a_{0}$ the Bohr radius, $E_{\mathrm{H}}$ the Hartree energy, and $m_{\mathrm{e}}$ the electron rest mass. This allows us to identify the different shells in the momentum spectra.

We have verified that resonant or even blue-detuned excitations of Rydberg atoms give rise to similar product-state distributions. In this case, however, the fraction of atoms which undergo a state-changing collision is substantially smaller as the

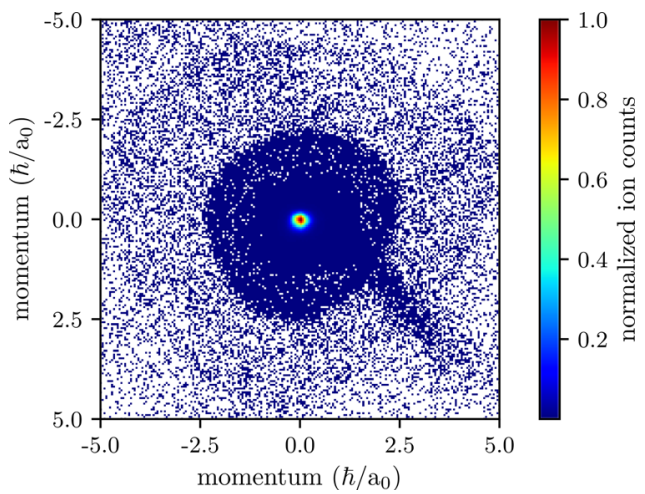

Fig. 3 Detector image resulting from the decay of 25P-ULRMs. The center of the plot consists of ions with vanishing transverse momentum, stemming mainly from photoionized ULRMs that have not undergone a statechanging collision. The two concentric circles at momenta 2.5 and $5.0 \mathrm{~h} / \mathrm{a}_{0}$ are due to the decay into the $22 \mathrm{Hy}$ and $21 \mathrm{Hy}$ hydrogenic manifolds. The feature in the lower right quadrant is due to technical issues of the detection unit. Sum of $10^{7}$ experimental runs, normalized to the maximum number of ion counts.

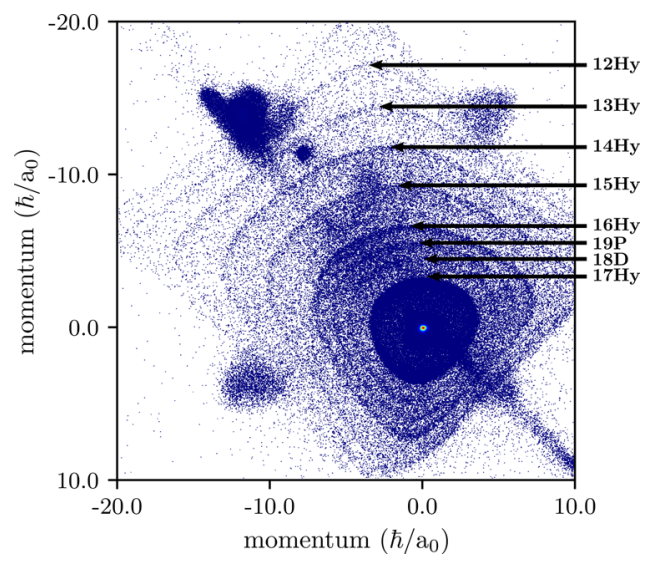

Fig. 4 Full detector image for state-changing collisions of 20P-ULRMs. Final states with principal quantum numbers down to $n=12$ are clearly visible. In addition, the decay to the 18D- and 19P-state can be observed, indicating the presence of low-I final states. The color code is the same as in Fig. 3. Deviations from the circular shape are due to design-related imperfections, causing a repulsion of the ions along the two diagonals. The artifacts in the corners of the detector plane are due to technical issues of the detection unit.

excited Rydberg atom has to find a collisional partner during the short evolution time until the ionization pulse is applied.

Considering a full detector image, as shown in Fig. 4 for the case of 20P-ULRMs, a large range of final states becomes apparent. Here, we cannot only observe the decay into manifolds as low as $n=12$; also states lying in between the manifolds are clearly visible. The deviations from the circular structure are artefacts caused by unshielded rods which hold the electrodes of the spectrometer and are set on a higher electric potential. As a consequence, the ions experience a Coulomb repulsion from four directions and the resulting momentum distribution appears to be pincushion-distorted. This especially applies to ions with large radial momenta moving at large distances from the spectrometer axis. Nevertheless, the final states can unambiguously be identified and evaluated. 


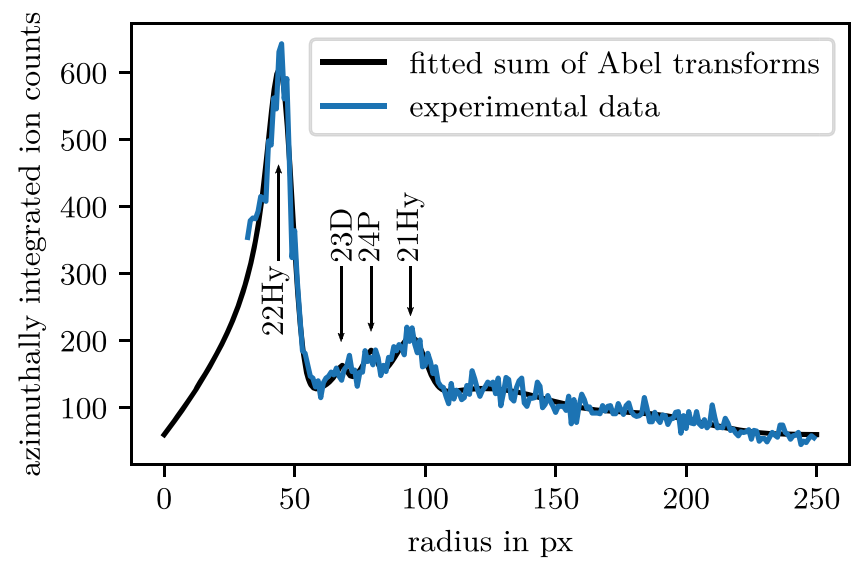

Fig. 5 Angular integrated radial profile of the momentum distribution shown in Fig. 3. The integration is performed over a circle segment, where the spectrum shows the least distortion. The center peak is omitted due to scaling purposes. Four peaks are visible. The two most pronounced peaks correspond to manifolds $22 \mathrm{Hy}$ and $21 \mathrm{Hy}$. In between, the 23D- and 24Pstates are visible. We do not see signatures of a decay to the 25S-state, which should appear between $22 \mathrm{Hy}$ and 23D. For higher radii, only the envelopes of the peaks are visible.

Analysis. We first concentrate our analysis on transitions from the initial state to the two lower-lying manifolds and the low- $l$ states in between. For a quantitative analysis of the momentum spectra we show the radial profile of the momentum distribution in Fig. 5. Since the outcome of our experiments is twodimensional projections of initially three-dimensional spherical shells, we make use of Abel transformations. To account for the finite thickness of the shells, which corresponds to the momentum uncertainty, we assume for the three-dimensional momentum distribution a Gaussian distribution of width $\sigma$ and amplitude $A$, which is shifted isotropically by the radius $R$ of the respective shell

$$
f(r, R, \sigma)=\frac{A}{\sqrt{2 \pi \sigma^{2}}} \exp \left(-\frac{(r-R)^{2}}{2 \sigma^{2}}\right),
$$

where $r^{2}=x^{2}+y^{2}+z^{2}$. The two-dimensional projection along the $z$-axis is then given by the Abel transform of Eq. (4)

$$
\begin{aligned}
F(\rho, R, \sigma) & =\int_{-\infty}^{\infty} f(r, R, \sigma) \mathrm{d} z \\
& =\int_{\rho}^{\infty} 2 \cdot f(r, R, \sigma) \cdot \frac{r}{\sqrt{r^{2}-\rho^{2}}} \mathrm{~d} r
\end{aligned}
$$

in which $\rho^{2}=x^{2}+y^{2}$.

Due to the azimuthal integration, the final fit function is given by $\rho \cdot F(\rho, R, \sigma)$. To account for the appearance of multiple peaks, we fit a sum of peaks to the data. For the radial profile shown in Fig. 5, for instance, we fitted a total of six peaks, of which four are sharp and clearly identifiable. The remaining two peaks are rather small and broad, which is mainly due to the continuously increasing distortion for higher transverse momenta and, hence, larger radii of the underlying momentum distribution. Thus, only the envelope of several peaks rather than individual peaks indicating the final states after the collision can be resolved. In summary, we find good agreement with our experimental data. From the fit, we extract the amplitudes, the momenta, and their uncertainties, encoded in $A, R$, and $\sigma$. Particularly, this allows us to evaluate the relative amplitude for each final state.

In addition to the two manifolds visible in Fig. 3, we can identify two more peaks stemming from the $23 \mathrm{D}$ and $24 \mathrm{P}$ final states. A systematic analysis for the initial quantum numbers $n \mathrm{P}$,

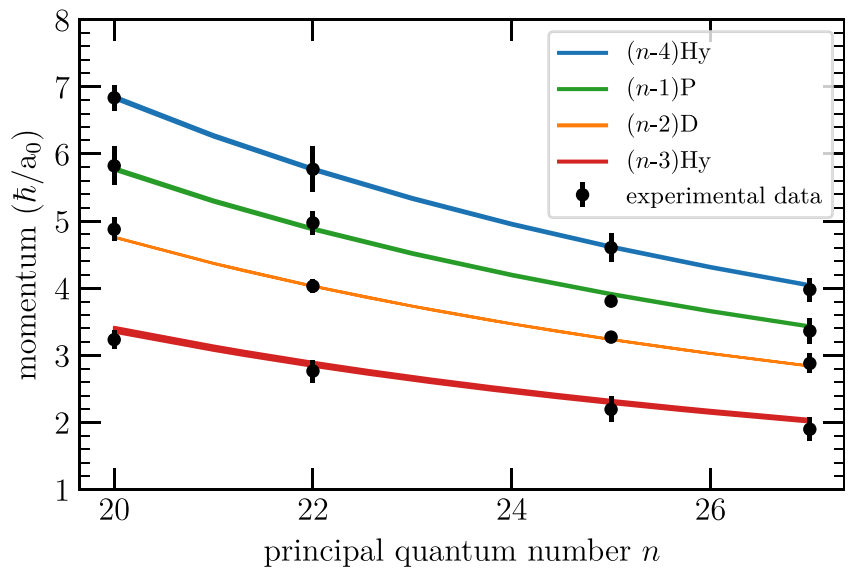

Fig. 6 Momenta resulting from state-changing collisions in dependence of the initial state. Depicted are the first four peaks, deduced from the fits to the respective radial profile (Fig. 5). The theoretically expected momenta are plotted as color-coded lines, where the thickness of the lines accounts for the momentum width (see text). The error bars indicate the momentum uncertainty as given by the fit parameter $\sigma$ of the Abel transform.

Table 1 Relative population of the final states down to the $(n-4) H y$ manifold and fraction of molecular ions $\mathbf{R b}_{2}^{+}$. All values are normalized to the sum of the detected atomic and molecular ions. The missing population is distributed over lower-lying states.

\begin{tabular}{lllllll} 
& $\boldsymbol{n P}$ & $(\boldsymbol{n}-\mathbf{3}) \mathbf{H y}$ & $(\boldsymbol{n}-\mathbf{2}) \mathbf{D}$ & $(\boldsymbol{n}-\mathbf{1 ) P}$ & $(\boldsymbol{n}-\mathbf{4}) \mathbf{H y}$ & $\mathbf{R b}_{\mathbf{2}}^{+}$ \\
\hline $27 \mathrm{P}$ & $89.7 \%$ & $1.5 \%$ & $0.1 \%$ & $0.01 \%$ & $0.4 \%$ & $3.6 \%$ \\
$25 \mathrm{P}$ & $92.1 \%$ & $1.3 \%$ & $0.1 \%$ & $0.1 \%$ & $0.4 \%$ & $2.6 \%$ \\
$22 \mathrm{P}$ & $74.2 \%$ & $4.0 \%$ & $0.2 \%$ & $0.3 \%$ & $0.6 \%$ & $8.2 \%$ \\
$2 \mathrm{OP}$ & $\mathbf{6 9 . 2 \%}$ & $3.3 \%$ & $0.3 \%$ & $0.6 \%$ & $1.5 \%$ & $2.3 \%$ \\
\hline
\end{tabular}

with $n \in\{20,22,25,27\}$ is shown in Fig. 6, where we plot the momenta of the fitted peaks in dependence of $n$. The expected momenta as calculated from the release energy are plotted as solid lines, where the thickness accounts for the spectral width of each final state including fine structure splitting and finite quantum defects of F- and G-states, which we include in the respective energetically close manifolds. In this procedure, we have introduced one global scaling factor to match experiment and theory, while the relative momenta remain conserved. The perfect agreement visible in Fig. 6 allows us to use this scaling factor as a calibration factor for all our momentum spectra.

In order to evaluate all detectable final states, we also include the molecular ions, which are created at short internuclear distances through associative ionization. Their signal is readily distinguished via their longer time of flight. The respective signals are then normalized to the total number of events $N_{\text {tot }}=N_{\mathrm{Rb}_{2}^{+}}+N_{\mathrm{Rb}^{+}}$. The results of this first part of the analysis are summarized in Table 1.

Inspecting Table 1, several trends are discernible. First, we note that the majority of events appear in the center of the momentum distribution. This is plausible as the signal in the center mainly consists of ions from long-living, photoionized ULRMs or from facilitated off-resonantly excited and photoionized Rydberg atoms. We find that these processes appear to become less likely for initial states with lower principal quantum numbers, in favor of state-changing collisions. Here, we particularly observed a pronounced decay to the $(n-3)$ hydrogenic manifold, which corroborates the experimental findings of Schlagmüller et al. ${ }^{9}$. 
Beyond that, our results reveal that the set of possible final states is not restricted to the first lower-lying hydrogenic manifold, but extends to numerous lower-lying states as well. Also the decay to isolated low angular momentum states, including P-states, is possible, which means that the observed collisions do not necessarily need to alter the angular quantum number. We therefore call these processes state-changing collisions rather than $l$-changing collisions as it is commonly referred to in literature (e.g., refs. ${ }^{30-32}$ ). Surprisingly, we could not find any indication of a decay into a final state with $l=0$. Evaluating our signal-to-noise ratio, such processes are at least suppressed by a factor of 10 .

The situation changes for the decay into hydrogenic manifolds, which appear to be more likely than the decay into isolated low- $l$ states. This is plausible as each manifold comprises a high amount of available final states. Moreover, the autoionization resonance width of Rydberg atoms becomes narrower for larger angular momentum quantum numbers, thus suppressing the formation of molecular ions ${ }^{33}$.

Of particular interest is also the ratio between state-changing collisions and $\mathrm{Rb}_{2}^{+}$formation. Evaluating the distribution listed in Table 1, a clear trend becomes apparent. When starting with a $27 \mathrm{P}$ ULRM, for instance, the occurrence of state-changing collisions is of the same order of magnitude as the formation of molecular ions (6.7\% compared to $3.6 \%)$. For lower initial states, however, we find a significantly enhanced incidence of state-changing collisions of up to $28.5 \%$ (for 20P-ULRMs), exceeding the fraction of molecular ions $(2.3 \%)$ by more than one order of magnitude.

Comparison with previous studies. These observations are new compared to previous studies ${ }^{9}$, where only the decay into one lower-lying manifold could be experimentally observed. However, there are also substantial differences concerning the experimental prerequisites. First of all, in ref. ${ }^{9}$, initial states with much higher principal quantum numbers $(n \geq 40)$ have been used. In this regime, enhanced state mixing gives rise to strong long-range couplings such that the dynamics predominantly occurs at large internuclear distances. A crucial role in this context plays the direct coupling between the butterfly PEC and the trilobite PEC (see also Fig. 1), which is assumed to be responsible for the decay into the next lower-lying manifold ${ }^{9}$. For initial states with lower principal quantum numbers, however, this coupling is strongly suppressed and cannot explain the observed redistribution of population. Instead, the probed dynamics is characterized by short-range interactions (see "Methods" section) rather than adiabatic couplings in the long-range part of the potential energy landscape and must therefore be clearly distinguished from the processes observed by Schlagmüller et al. ${ }^{9}$.

Another relevant difference in this connection is the number of ground state atoms inside the wave function of the Rydberg electron, which could considerably influence the collision process. Due to the low principal quantum numbers of the initial states used in our experiment, we expect this number, on average, to be well below 1 . In ref. ${ }^{9}$, however, not only the principal quantum numbers of the initial states but also the densities of the atomic sample are higher, which results in an average of up to 1000 ground state atoms within the orbit of the Rydberg electron, bound to one single Rydberg core.

Apart from that, it should be noted that not only the principal quantum numbers but also the angular momentum quantum numbers of the initial states are different. In fact, instead of $n \mathrm{P}$ states, the experiments performed by Schlagmüller et al. ${ }^{9}$ rely on $n S$-states. Looking at the molecular PECs in Fig. 1, it becomes obvious that the initial dynamics starting from those states is quite different due to the involved quantum defects.

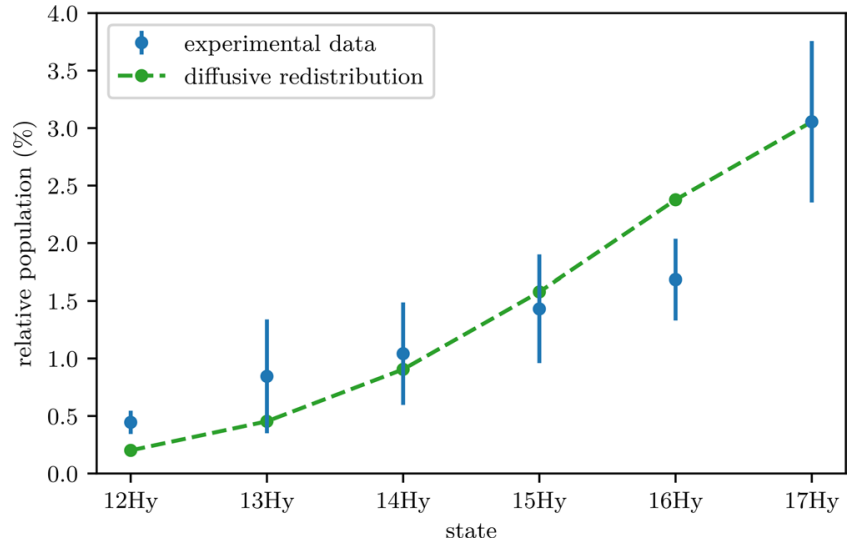

Fig. 7 Distribution of the final state population upon state-changing collisions starting from 20P-ULRMs. The data are normalized to the sum of events that ended up in the respective states. The diffusive model (see text) is shown as green dashed line and reproduces the experimental data well. The error bars indicate systematic uncertainties of the determined populations.

Our approach is especially sensitive to small signals at high momenta, thus facilitating the detection of lower-lying states due to a high signal-to-noise ratio. Only this way, it is possible to resolve the redistribution of population as a result of statechanging collisions. Due to major differences concerning both the experimental approach and the probed dynamics, our findings are distinct from previously reported results presented in ref. ${ }^{9}$.

Short-range dynamics. We now turn our attention to the details of the population distribution between the final states. In Fig. 4, one can clearly see the decay into six hydrogenic manifolds when starting with a 20P-ULRM. For simplicity, we restricted ourselves to the manifolds only and neglect low- $l$ states, which are, by comparison, substantially suppressed. The measured population in each manifold is shown in Fig. 7. One can clearly see a continuous decrease of the signal, with substantial weight even at the lowest detectable quantum number $(n=12)$. In order to explain the wide distribution of final states, we have to look at the microscopic details of the molecular PEC couplings. In ref. ${ }^{9}$, it was argued that the decay into the lower-lying manifold is due to a direct coupling of the butterfly PEC with the trilobite PEC. For an initial $n \mathrm{P}$-state with low principal quantum number, the direct coupling between the butterfly state and the trilobite state becomes small and cannot fully explain the strong decay into the next lower-lying manifold and even less the distribution among the other final states observed in our experiment. Considering couplings within the long-range part of the PEC landscape only is therefore not expedient. In fact, to understand this process one has to look in more detail at the molecular dynamics at short internuclear distances.

Following ref. ${ }^{34}$, inelastic collisions between Rydberg atoms and ground state atoms can often be subdivided into three phases: (1) the approach of the particles until they strongly couple to each other, (2) the formation of a Rydberg quasi-molecular complex at short internuclear distances, and (3) the outcome of the collision, a state-change followed by dissociation or associative ionization. Applying this principle to the present case, the ionic core of the Rydberg atom and the neutral atom first approach each other, following the long-range PECs. When the internuclear distance has reached values $\lesssim 30 a_{0}$, the subsequent dynamics can be described in the framework of a Rydberg diatomic quasi-molecular complex. This complex consists of two positively charged $\mathrm{Rb}^{+}$cores, a generalized valence electron stemming from the ground state atom 


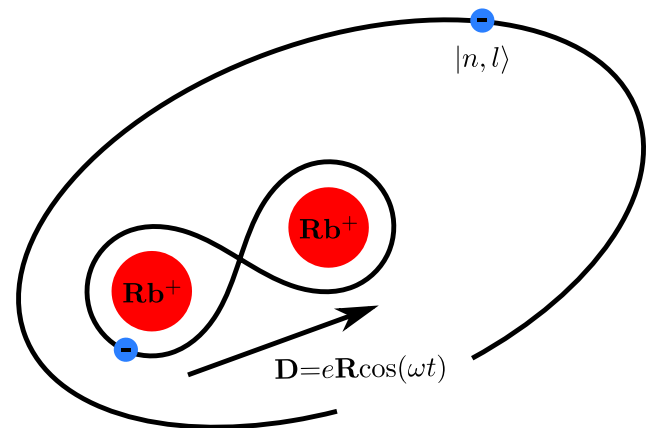

Fig. 8 Rydberg quasi-molecular diatomic complex. The short-range dynamics of the collision between a Rydberg atom and a ground state atom can be described in the framework of a short-lived Rydberg quasi-molecular complex, where tunneling of the inner valence electron between the two ionic cores leads to an oscillating dipole moment $\mathrm{D}$. As a result, the oscillating dipole induces transitions of the Rydberg electron.

and a Rydberg electron, which is shared by the molecular ionic core $\mathrm{Rb}_{2}^{+}$(see Fig. 8). At such short internuclear distances, the so-called dipole resonant mechanism ${ }^{35,36}$ becomes active. When the ionic core and the ground state atom approach each other, the valence electron starts to tunnel between the two ionic cores. This leads to an oscillating internal dipole moment $\mathbf{D}(t)=e \mathrm{R} \cos (\omega \mathrm{t})$ of the quasi-molecule, where $\mathbf{R}$ denotes the distance of the two ionic cores, $\omega=\Delta(R)$ is the splitting between the gerade and ungerade wave function of the inner valence electron, and $e$ is the elementary charge. The periodic potential leads to an oscillating electric field with dipolar radiation characteristics, which can induce transitions of the Rydberg electron. In this semiclassical picture of the collision process, the varying distance between the ionic core and the ground state atom leads to a time-varying oscillation frequency. It is therefore not surprising that these mechanisms can induce many transitions within and between different Rydberg manifolds.

To illustrate these mechanisms, we show in Fig. 9 a simplified version of the PECs at short internuclear distances. All final states of the collision when starting with 20P-ULRMs are highlighted. Considering only the molecular ion, $\mathrm{Rb}_{2}^{+}$, we have two PECs, $U_{\mathrm{g}}(R)$ and $U_{\mathrm{u}}(R)$ for the terms ${ }^{2} \Sigma_{\mathrm{g}}^{+}$and ${ }^{2} \Sigma_{\mathrm{u}}^{+}$. The ${ }^{2} \Sigma_{\mathrm{g}}^{+}$state is the ground state of the molecular ion and forms a deep potential well. The PEC labeled as ${ }^{2} \Sigma_{\mathrm{u}}^{+}$, however, is predominantly repulsive with a shallow attractive section at larger internuclear distances. The energy difference between both states $\hbar \times \Delta(R)$ determines the oscillation frequency of the electron when tunneling between the two ionic cores.

The inclusion of the Rydberg electron is now done in a trivial way by only taking care of its binding energy. This results in copies of the PECs, shifted by the binding energy of the Rydberg electron

$$
U_{\mathrm{g}, \mathrm{u}}^{(n, l)}(R)=U_{\mathrm{g}, \mathrm{u}}(R)+E_{\mathrm{bind}}(n, l) .
$$

All other couplings, such as the spin-spin interaction, fine structure splitting, hyperfine structure effects, or the exchange interaction between the two electrons, are so small that they are negligible on the energy scale given by $\hbar \times \Delta(R)$. The relevant molecular symmetry for all PECs is therefore the gerade and ungerade one of the molecular ionic core.

The PECs in Fig. 9 show a plenitude of crossings between the gerade and ungerade states. Due to the resonant dipole mechanism, all of these are avoided and the molecule can undergo transitions between the different PECs, resulting in an effective redistribution of the populations during the collision.

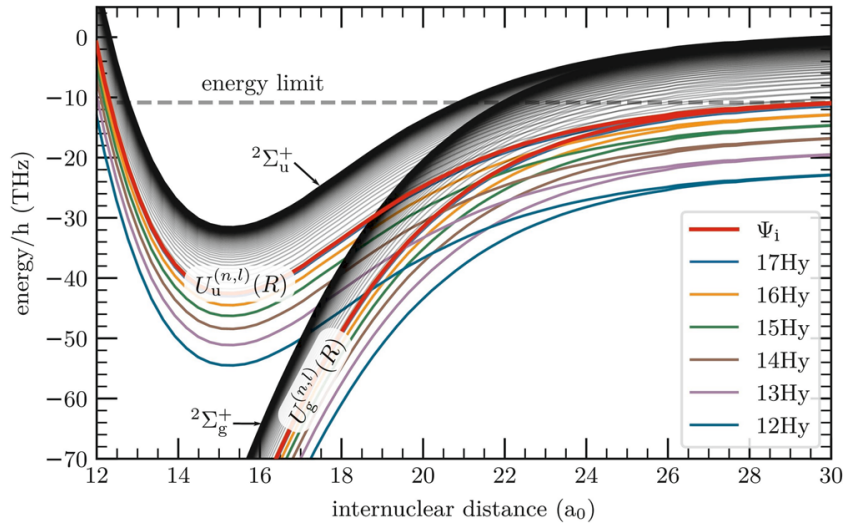

Fig. 9 Potential energy landscape of a Rydberg quasi-molecular complex. The molecular PECs split into two branches, which belong to the ${ }^{2} \Sigma_{g}^{+}$and ${ }^{2} \Sigma_{\mathrm{u}}^{+}$components of the quasi-molecular ion. The highest-lying PEC of each branch belongs to $U_{\mathrm{g}}(R)\left(U_{\mathrm{u}}(R)\right)$ of the molecular ion. All other PECs $U_{g, u}^{(n, l)}(R)$ are shifted by the binding energy of the Rydberg electron. For simplicity, we restrict the plot to hydrogenic manifolds only. The multicolored PECs are relevant for the description of the state-changing collision process when starting with 20P-ULRMs (see also Fig. 4). When the frequency of the oscillating dipole $\omega=\Delta(R)$ exceeds the binding energy of the Rydberg electron, the complex may undergo associative ionization. The gray dashed line indicates the energy limit as defined by the asymptotic potential of the initial state $\Psi_{\mathrm{i}}$.

It is instructive to look at the coupling strength between the PECs. Based on the semiclassical model introduced above, we can make the following estimate: we calculated the electric field $E(t)$ induced by the oscillating internal dipole at the classical radius of the Rydberg electron's orbit. We further simplify the system by assuming that the resulting electric field is spatially homogeneous across the Rydberg electron wave function. Together with the typical transition matrix elements between neighboring Rydberg states, given by $e a_{0} n^{2}$, we get the coupling strength $\hbar \Omega$. The resulting values of $\Omega$ are in the Terahertz range and are therefore comparable to or even higher than the energetic distance of adjacent manifolds. This complicates the molecular dynamics at short internuclear distances even further, as the coupling cannot be considered as a small perturbation to the PECs. Consequently, surface hopping model ${ }^{37}$ are expected to be not applicable (see also ref. ${ }^{38}$ ).

Diffusive-like redistribution of population. To account for this strong mixing of the PECs, we adopt an effective model from ref. ${ }^{38}$, where the redistribution of population between the Rydberg states is the consequence of a diffusive motion of the Rydberg electron in microwave fields ${ }^{38}$. This approach has been successfully employed for the description of collisional or thermal ionization processes $36,38-44$. As the physical mechanisms in a state-changing collision are the same, it is applicable in our case as well. The stochastic motion of the Rydberg electron is described by a diffusion equation

$$
\frac{\partial}{\partial t} \Phi(n, t)=\mathcal{D} \frac{\partial^{2}}{\partial n^{2}} \Phi(n, t),
$$

where $\Phi(n, t)$ is the distribution of a Rydberg electron in the space of principal quantum numbers $n$ and $\mathcal{D}$ is the diffusion coefficient. Prior to the redistribution, the main principal quantum number of the initial state is given by $n_{\mathrm{i}}$. Due to the mixing of the butterfly state, we assume the population to be initially in a state with the principal quantum number $n_{\mathrm{i}}=18$ with probability $p_{\mathrm{i}}$. We then solve Eq. (7) using $\mathcal{D}$ as a fit parameter. The results are 
shown in Fig. 7 and show good agreement with our experimental results, thus confirming a diffusive-like redistribution between the final states.

One might wonder, why the diffusive model describes the experimental observation so well, given its simplicity. In fact, the exact microscopic ingredients are much more complex since the diffusion coefficient $\mathcal{D}$ in Eq. (7) is not necessarily a constant $^{34}$ and the oscillating electric dipole radiation field is far from being homogeneous. Moreover, we have completely ignored the $n-1$ angular momentum states of each manifold, which all have different matrix elements, and that the initial butterfly state is made up by a large number of angular momentum states.

However, when so many different initial angular momentum states and so many couplings with different strength between a plenitude of PECs contribute to the system dynamics, a diffusivelike behavior might be, after all, just the most likely one. Our results might therefore be interpreted as a manifestation of the central limit theorem.

\section{Discussion}

In this manuscript, we demonstrate that inelastic collisions between Rydberg and ground state atoms can result in a large range of final states. We give evidence for the decay into low angular momentum states over a large range of principal quantum numbers. We also find pronounced decay into many lower-lying hydrogenic manifolds with substantial weight. The distribution among the manifolds suggests a diffusive-like redistribution between the Rydberg states at short internuclear distances. We give a simplified explanation of this behavior in terms of redistribution of Rydberg states in microwave fields. An ab initio quantum-chemical treatment of the total collision process is a challenging task, given the different interaction mechanisms at short and large internuclear distances. Nevertheless, our results help to model parts of the collisions more accurately. Our results also have implications for the modeling of inelastic processes in many-body Rydberg systems. In the future, it will be interesting to look for effects of alignment in the initial state, where the resulting momentum distribution after the collisions becomes anisotropic. This would exploit the full 3D imaging capability of our momentum spectrometer. We expect that this development will also allow for the study of other dynamical processes in Rydberg systems such as Rydberg-Rydberg dynamics, the direct measurement of the momentum distribution of Rydberg molecules and the study of other exotic Rydberg matter, such as heavy Rydberg systems $^{45}$

\section{Methods}

Ultralong-range Rydberg molecules. ULRMs are bound states between a Rydberg atom and at least one ground state atom. The binding results from low-energy scattering between the Rydberg electron and the ground state atom and can be expressed in the formalism of a Fermi pseudopotential ${ }^{1,24,46-49}$

$$
\begin{aligned}
V_{\mathrm{e}, \mathrm{g}}(|r-R|)= & V_{s}(|r-R|)+V_{p}(|r-R|) \\
= & 2 \pi a_{s}[k(R)] \delta(r-R) \\
& +6 \pi a_{p}[k(R)] \overleftarrow{\nabla} \delta(r-R) \vec{\nabla}
\end{aligned}
$$

where $\mathbf{r}$ is the position of the Rydberg electron and $\mathbf{R}$ the position of the ground state atom with respect to the Rydberg ionic core. The first term describes s-wave interactions, which dominate at sufficiently large internuclear distances. At smaller internuclear distances of a few hundred Bohr radii, the p-wave scattering interaction comes into play. In case of alkali atoms, it equips the potential energy landscape with an attractive potential, associated with the so-called butterfly $\mathrm{PECs}^{24-26}$, which arise from an underlying $\mathrm{p}$-wave shape resonance.

Besides the electron-atom scattering, one also has to account for the attractive long-range interaction between the ionic core and the polarizable ground state atom, which is given by

$$
V_{c}, g(R)=-\alpha /\left(2 R^{4}\right)
$$

The effective Hamiltonian for the Rydberg electron therefore reads

$$
\mathcal{H}=\mathcal{H}_{0}(r)+V_{\mathrm{c}}, \mathrm{g}(R)+V_{\mathrm{e}}, \mathrm{g}(|\mathrm{r}-\mathrm{R}|),
$$

where $\mathcal{H}_{0}(r)$ is the Hamiltonian of the bare Rydberg atom. By diagonalizing this Hamiltonian in a finite set of basis states Born-Oppenheimer PECs can be deduced. Since the energy shift due to $V_{s}(|\mathrm{r}-\mathrm{R}|)$ is proportional to the electron probability density at the position of the perturber, the PECs are oscillatory functions of $R$ with localized wells at large separations, which can support closely spaced bound vibrational states.

\section{Data availability}

The data that support the findings of this study are available from the corresponding author upon reasonable request.

Received: 16 February 2021; Accepted: 1 June 2021; Published online: 23 June 2021

\section{References}

1. Fermi, E. Sopra lo spostamento per pressione delle righe elevate delle serie spettrali. Il Nuovo Cimento (1924-1942) 11, 157 (1934).

2. Rolston, S. L. \& Roberts, J. L. Ultracold neutral plasmas. The Expanding Frontier Of Atomic Physics pp. 73-82 (2003).

3. Klyucharev, A. N., Bezuglov, N. N., Mihajlov, A. A. \& Ignjatović, L. M. Influence of inelastic Rydberg atom-atom collisional process on kinetic and optical properties of low-temperature laboratory and astrophysical plasmas. $J$. Phys.: Conf. Ser. 257, 012027 (2010).

4. Shaffer, J. P., Rittenhouse, S. T. \& Sadeghpour, H. R. Ultracold Rydberg molecules. Nat. Commun. 9, 1965 (2018).

5. Urban, E. et al. Observation of Rydberg blockade between two atoms. Nat Phys. 5, 110 (2009).

6. Weber, T. et al. Mesoscopic Rydberg-blockaded ensembles in the superatom regime and beyond. Nat. Phys. 11, 157 (2015).

7. Amthor, T., Giese, C., Hofmann, C. S. \& Weidemüller, M. Evidence of antiblockade in an ultracold Rydberg gas. Phys. Rev. Lett. 104, 013001 (2010).

8. Barredo, D. et al. Coherent excitation transfer in a spin chain of three Rydberg atoms. Phys. Rev. Lett. 114, 113002 (2015).

9. Schlagmüller, M. et al. Ultracold chemical reactions of a single Rydberg atom in a dense gas. Phys. Rev. X 6, 031020 (2016).

10. Goldschmidt, E. A. et al. Anomalous broadening in driven dissipative Rydberg systems. Phys. Rev. Lett. 116, 113001 (2016).

11. Wolf, S. \& Helm, H. Ion-recoil momentum spectroscopy in a laser-cooled atomic sample. Phys. Rev. A 62, 043408 (2000).

12. Van der Poel, M., Nielsen, C., Gearba, M.-A. \& Andersen, N. Fraunhofer diffraction of atomic matter waves: electron transfer studies with a laser cooled target. Phys. Rev. Lett. 87, 123201 (2001).

13. Turkstra, J. et al. Recoil momentum spectroscopy of highly charged ion collisions on magneto-optically trapped Na. Phys. Rev. Lett. 87, 123202 (2001).

14. Flechard, X., Nguyen, H., Wells, E., Ben-Itzhak, I. \& DePaola, B. Kinematically complete charge exchange experiment in the cs $++\mathrm{r}$ b collision system using a mot target. Phys. Rev. Lett. 87, 123203 (2001).

15. Nguyen, H., Fléchard, X., Brédy, R., Camp, H. \& DePaola, B. Recoil ion momentum spectroscopy using magneto-optically trapped atoms. Rev. Sci. Instrum. 75, 2638 (2004).

16. Blieck, J. et al. A new magneto-optical trap-target recoil ion momentum spectroscopy apparatus for ion-atom collisions and trapped atom studies. Rev. Sci. Instrum. 79, 103102 (2008).

17. DePaola, B., Morgenstern, R. \& Andersen, N. Motrims: magneto-optical trap recoil ion momentum spectroscopy. Adv. At., Mol., Opt. Phys. 55, 139 (2008).

18. Schuricke, M. et al. Strong-field ionization of lithium. Phys. Rev. A 83, 023413 (2011).

19. Fischer, D. et al. Ion-lithium collision dynamics studied with a laser-cooled in ring target. Phys. Rev. Lett. 109, 113202 (2012).

20. Götz, S. et al. Versatile cold atom target apparatus. Rev. Sci. Instrum. 83, 073112 (2012).

21. Hubele, R. et al. Electron and recoil ion momentum imaging with a magnetooptically trapped target. Rev. Sci. Instrum. 86, 033105 (2015).

22. Li, R. et al. Recoil-ion momentum spectroscopy for cold rubidium in a strong femtosecond laser field. J. Instrum. 14, P02022 (2019).

23. Fey, C., Hummel, F. \& Schmelcher, P. Ultralong-range Rydberg molecules Mol. Phys. 118, e1679401 (2020).

24. Hamilton, E. L., Greene, C. H. \& Sadeghpour, H. Shape-resonance-induced long-range molecular Rydberg states. J. Phys. B: At., Mol. Opt. Phys. 35, L199 (2002). 
25. Chibisov, M., Khuskivadze, A. \& Fabrikant, I. Energies and dipole moments of long-range molecular Rydberg states. J. Phys. B: At., Mol. Opt. Phys. 35, L193 (2002).

26. Niederprüm, T. et al. Observation of pendular butterfly Rydberg molecules. Nat. Commun. 7, 1 (2016).

27. Markert, F. et al. AC-Stark shift and photoionization of Rydberg atoms in an optical dipole trap. New J. Phys. 12, 113003 (2010).

28. Gabbanini, C. Assessments of lifetimes and photoionization cross-sections at $10.6 \mu \mathrm{m}$ of nd Rydberg states of rb measured in a magneto-optical trap. Spectrochim. Acta Part B: At. Spectrosc. 61, 196 (2006).

29. Wiley, W. \& McLaren, I. H. Time-of-flight mass spectrometer with improved resolution. Rev. Sci. Instrum. 26, 1150 (1955).

30. Higgs, C., Smith, K., Dunning, F. \& Stebbings, R. A study of 1 changing in xe (nf)-neutral collisions at thermal energies. J. Chem. Phys. 75, 745 (1981).

31. Matsuzawa, M. Validity of the impulse approximation in Rydberg-neutral collisions. J. Phys. B: At. Mol. Phys. 17, 795 (1984).

32. Lebedev, V. S. \& Fabrikant, I. I. Semiclassical calculations of the 1-mixing and n, l-changing collisions of Rydberg atoms with rare-gas atoms. J. Phys. B: At., Mol. Opt. Phys. 30, 2649 (1997).

33. Gallagher, T. F. Rydberg Atoms, Vol. 3 (Cambridge Univ. Press, 2005).

34. Dimitrijević, M. S., Srećković, V. A., Zalam, A. A., Bezuglov, N. N. \& Klyucharev, A. N. Dynamic instability of Rydberg atomic complexes. Atoms 7, 22 (2019).

35. Smirnov, V. \& Mihajlov, A. Nonelastic collisions involving highly excited atoms. Opt. Spectrosc. 30, 525 (1971)

36. Mihajlov, A., Srećković, V., Ignjatović, L. M. \& Klyucharev, A. The chemiionization processes in slow collisions of Rydberg atoms with ground state atoms: mechanism and applications. J. Cluster Sci. 23, 47 (2012).

37. Belyaev, A. K., Lasser, C. \& Trigila, G. Landau-zener type surface hopping algorithms. J. Chem. Phys. 140, 224108 (2014).

38. Bezuglov, N. et al. Diffusion ionization of the Rydberg diatomic quasimolecular complex formed upon collisions of rubidium atoms. Opt. Spectrosc. 95, 515 (2003).

39. Duman, E. \& Shmatov, I. Ionization of highly excited atoms in their own gas. Sov. Phys. JETP 51, 1061 (1980).

40. Janev, R. \& Mihajlov, A. Resonant ionization in slow-atom-Rydberg-atom collisions. Phys. Rev. A 21, 819 (1980).

41. Mihajlov, A. \& Janev, R. Ionisation in atom-Rydberg atom collisions: ejected electron energy spectra and reaction rate coefficients. J. Phys. B: At. Mol. Phys. 14, 1639 (1981).

42. Bezuglov, N. et al. Analysis of Fokker-Planck type stochastic equations with variable boundary conditions in an elementary process of collisional ionization. Opt. Spectrosc. 91, 19 (2001).

43. Bezuglov, N., Borodin, V., Eckers, A. \& Klyucharev, A. A quasi-classical description of the stochastic dynamics of a Rydberg electron in a diatomic quasi-molecular complex. Opt. Spectrosc. 93, 661 (2002).

44. Miculis, K. et al. Collisional and thermal ionization of sodium Rydberg atoms: Ii. theory for ns, $\mathrm{np}$ and $\mathrm{nd}$ states with $\mathrm{n}=5-25$. J. Phys. B: At., Mol. Opt. Phys. 38, 1811 (2005).

45. Hummel, F., Schmelcher, P., Ott, H. \& Sadeghpour, H. R. An ultracold heavy Rydberg system formed from ultra-long-range molecules bound in a stairwell potential. New J. Phys. 22, 063060 (2020)

46. Omont, A. On the theory of collisions of atoms in Rydberg states with neutral particles. J. de Phys. 38, 1343 (1977).

47. Greene, C. H., Dickinson, A. \& Sadeghpour, H. Creation of polar and nonpolar ultra-long-range Rydberg molecules. Phys. Rev. Lett. 85, 2458 (2000).
48. Fey, C., Kurz, M., Schmelcher, P., Rittenhouse, S. T. \& Sadeghpour, H. R. A comparative analysis of binding in ultralong-range Rydberg molecules. New J. Phys. 17, 055010 (2015).

49. Eiles, M. T. \& Greene, C. H. Hamiltonian for the inclusion of spin effects in long-range Rydberg molecules. Phys. Rev. A 95, 042515 (2017).

\section{Acknowledgements}

The authors thank the group of Reinhard Dörner (Goethe University of Frankfurt) for helpful discussions regarding the design of the MOTRIMS apparatus and Dominik Arnold for constructing the spectrometer. The authors thank Cihan Sahin for his help on setting up the experimental apparatus. The authors also thank Bergmann Messgeräte Entwicklung KG for the excellent Pockels cell driver and the invaluable technical support. The authors acknowledge fruitful discussions with Peter Schmelcher and Frederic Hummel (University of Hamburg). The authors acknowledge financial support by the German Research Foundation (Deutsche Forschungsgemeinschaft) within the priority program 'Giant Interactions in Rydberg Systems' (DFG SPP 1929 GiRyd, project no. 316211972).

\section{Author contributions}

P.G., M.A., and D.F. performed the experiment and analyzed the data. H.O. supervised the experiment. P.G. prepared the manuscript. All authors developed the theoretical model and contributed to the data interpretation and manuscript preparation.

\section{Funding}

Open Access funding enabled and organized by Projekt DEAL.

\section{Competing interests}

The authors declare no competing interests.

\section{Additional information}

Correspondence and requests for materials should be addressed to H.O.

Peer review information Nature Communications thanks Michał Tomza and the other, anonymous, reviewer(s) for their contribution to the peer review of this work.

Reprints and permission information is available at http://www.nature.com/reprints

Publisher's note Springer Nature remains neutral with regard to jurisdictional claims in published maps and institutional affiliations.

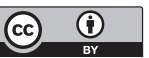

Open Access This article is licensed under a Creative Commons Attribution 4.0 International License, which permits use, sharing, adaptation, distribution and reproduction in any medium or format, as long as you give appropriate credit to the original author(s) and the source, provide a link to the Creative Commons license, and indicate if changes were made. The images or other third party material in this article are included in the article's Creative Commons license, unless indicated otherwise in a credit line to the material. If material is not included in the article's Creative Commons license and your intended use is not permitted by statutory regulation or exceeds the permitted use, you will need to obtain permission directly from the copyright holder. To view a copy of this license, visit http://creativecommons.org/ licenses/by/4.0/.

(c) The Author(s) 2021 